\title{
Corela
}

Cognition, représentation, langage

HS-6 | 2007

Cognition, discours, contextes

\section{Discours et cognition : les prédiscours entre cadres internes et environnement extérieur}

\section{Marie-Anne Paveau}

\section{(2) OpenEdition}

\section{Journals}

Édition électronique

URL : http://journals.openedition.org/corela/1550

DOI : $10.4000 /$ corela. 1550

ISSN : $1638-573 \mathrm{X}$

Éditeur

Cercle linguistique du Centre et de I'Ouest - CerLICO

Référence électronique

Marie-Anne Paveau, « Discours et cognition : les prédiscours entre cadres internes et environnement extérieur », Corela [En ligne], HS-6 | 2007, mis en ligne le 01 novembre 2007, consulté le 19 avril 2019. URL : http://journals.openedition.org/corela/1550; DOI : 10.4000/corela.1550

Ce document a été généré automatiquement le 19 avril 2019

\section{(c) (1) (3) (-)}

Corela - cognition, représentation, langage est mis à disposition selon les termes de la licence Creative Commons Attribution - Pas d'Utilisation Commerciale - Partage dans les Mêmes Conditions 4.0 International. 


\title{
Discours et cognition : les prédiscours entre cadres internes et environnement extérieur
}

\author{
Marie-Anne Paveau
}

Le cerveau des autres me semble admirablement
organisé : on jurerait qu'il contient une infinité de
petites boîtes étiquetées Situation internationale
(deux boîtes), Bourse, Cancer, Chambre (deux boîtes),
Potins (quatre boîtes), Sécurité sociale, Cinéma (trois
boîtes), Mariages, Théâtre (trois boîtes). [...] La
maîtresse de maison, ou toute autre, n'a qu'un mot
à jeter sur la nappe, ou dans ce rond stupide que
dix fauteuils délimitent, et les voilà qui extraient le
contenu de leurs petites boîtes, aussi sûrement
qu'un paléographe lirait un parchemin (P. Daninos,
1952 , Sonia, les autres et moi).

\section{Introduction}

1 L'analyse du discours, désormais dictionnarisée (Charaudeau, Maingueneau 2002, Détrie et al., 2001, Colletta, Nuchèze 2002), synthétisée (Maingueneau (dir.) 2005, Paveau, Rosier 2005, Mazière 2005), et largement utilisée comme méthodologie d'analyse dans les sciences humaines et sociales (information-communication, sociologie, science politique, voir Temmar, Bonnafous (dir.) 2007), est à la recherche d'un nouveau souffle théorique. De leur côté les sciences cognitives ont depuis les années 1980-1990 intégré les questions du contexte et de la culture (par exemple Fauconnier, Turner, Sweetser, Verhagen, Achard-Bayle, Nyckees) et s'ancrent actuellement, en France tout du moins, dans une histoire intellectuelle qui reconnaît désormais la place qui a été faite aux activités 
mentales dans la modélisation de la production langagière (Valette 2006, Moirand icimême).

La rencontre semble inévitable et sur le terrain encore étroit et nouveau que constitue cette articulation, certaines recherches tentent de rendre compte de la dimension cognitive dans le discursif et inversement. Plusieurs directions se dessinent en France actuellement : une approche sémantique en texte-discours ${ }^{1}$ qui reprend la question de la référenciation dans la perspective de la cognition culturelle (Achard-Bayle 2005, 2006), à travers la question du nom propre par exemple (Cislaru 2005); une approche inscrite dans l'analyse du discours dite française $e^{2}$ qui travaille les formes langagières de la mémoire et de l'allusion (Moirand 2003, 2004); une articulation discours-cognition mettant à contribution la phénoménologie de la perception et la cognition sociale (Paveau 2006a, 2007a et b). Dans le monde anglo-saxon, l'articulation «discourse and cognition» a été posée dans les années 1990 (Edwards 1997) et récemment synthétisée dans un numéro de Discourse Studies dirigé par Van Dijk (2006). C'est donc peu de dire que les approches cognitives du texte et du discours sont désormais engagées et bien engagées : un espace théorique fort s'est ouvert, et les disciplines textuelles et discursives ont tout intérêt à venir y puiser un enrichissement de leurs fondations et un renforcement de leurs méthodes.

3 C'est dans cette perspective que j'aborde l'articulation discours et cognition dans cet article. Je commencerai par faire le point sur ce que j'appelle le tournant cognitif de l'analyse du discours, dans les domaines anglo-saxon et français, avant de présenter le concept de prédiscours, qui est ma proposition théorique pour traiter l'articulation discours-cognition. J'approfondirai ensuite la notion de technologie discursive, qui permet de penser l'activité de production langagière en rapport avec l'environnement non humain, en particulier objectal et technique.

\section{Le tournant cognitif de l'analyse du discours}

4 Parler de "tournant cognitif » est un peu stéréotypé mais cela permet de nommer un ensemble de travaux actuels qui, sans être forcément connectés, reposent tous sur l'appel aux données de la cognition pour (re)penser le fonctionnement des textes et des discours. Cet appel implique une interrogation sur la nature et le fonctionnement du contexte.

\subsection{L'épineuse question du contexte dans les disciplines texte- discours}

On sait depuis longtemps que la notion de contexte est indispensable à l'appareillage théorique de celui qui souhaite rendre compte des productions verbales : bien que l'appel au contexte ne se soit naturalisé dans les linguistiques texte-discours qu'à partir des années 1960 (pour une synthèse : Achard-Bayle (coord.) 2006), la dimension théorique est très présente dans les travaux des chercheurs qui, dans les années 1920, proposent les premières approches du langage en situation ${ }^{3}$ : chacun à leur manière, sous des étiquettes différentes et avec des approches disciplinaires variées, Sapir (Linguistique en 1921), Malinovski (Les argonautes du Pacifique en 1922, Mœurs et coutumes des Mélanésiens en 1933) et Bakhtine (Esthétique et théorie du roman en 1924, Le marxisme et la philosophie du langage 
en 1929) conditionnent toute étude texte-discours à la prise en compte du contexte culturel, social immédiat ou plus large, historique et axiologique.

Malgré l'ancienneté de la notion, et peut-être à cause d'elle, la notion de contexte reste insuffisante pour rendre compte de la production discursive des individus dans le monde, sans doute parce qu'elle reçoit une interprétation trop étroite (appel aux paramètres énonciatifs ou même aux identités et rôles sociaux) ou trop large (les "situations sociales » sont souvent assimilées à des ères sociales comme le travail, les loisirs ou des situations de sociabilité, et les «conditions de production » sont souvent alléguées sans être véritablement décrites). Mazière 2005 remarque que le contexte est très peu défini dans les deux dictionnaires Charaudeau, Maingueneau 2002 et Détrie et al. 2001. Je note qu'il est en revanche très bien défini dans le Guide terminologique de Colletta et Nuchèze (2002), sur quatre pages comprenant les origines, les différentes versions, les dérivés, les néologismes (to-texte par exemple).

7 Pourquoi cette relative pauvreté de la notion, ou son appauvrissement historique ? Il y a plusieurs réponses à cette question. D'abord parce que, comme l'a montré Mazière 2005, l'analyse du discours dite "à la française » a nié le rapport au contexte en faisant du discours son propre contexte; ensuite parce que des notions à la fois concurrentes, synonymes, et englobantes, ont tenu lieu de définition du contexte par dénomination : la notion d'interaction par exemple, que ce soit via l'interactionnisme américain ou le «revival » du dialogisme de Bakhtine dans les années 1990, est devenue une sorte de passage obligé, dans les représentations légitimo-institutionnelles de tout linguiste qui travaille sur le texte-discours; enfin, et je pense qu'il s'agit de la réponse la plus pertinente, parce qu'il y a du paradoxe dans la notion : «Le contexte est un objet linguistique paradoxal ; il est à la fois partout et mal identifié » (Achard-Bayle 2006:5), et de conclure sur l'aspect incontournable mais en même temps toujours contourné de la notion quelque peu réduite à une fonction décorative : «Bref, ce contexte qui a massivement investi les recherches linguistiques (et dans un grand nombre de ses orientations actuelles : énonciatives, discursives, textuelles, cognitives, socio-, psycho-, ethnolinguistiques) semble condamné à jouer le rôle ou tenir la place que lui assigne sa définition même: l'«arrière-plan »... (2006: 5). Comme le signale très justement Chabrolle-Cerretini, l'étude du contexte " demeure une entreprise de grande envergure " qui nécessite la convocation de paramètres multiples (2006: 97).

8 L'appel aux données de la cognition, parce qu'il oblige le chercheur à repenser la notion de contexte, semble à même de fournir des éléments et des regards nouveaux sur cette réalité complexe. Le domaine "discourse and cognition» étant actuellement occupé par Van Dijk, il est nécessaire de présenter son approche.

\section{2. «Mental models », une version faible de la cognition}

Van Dijk propose depuis les années 1980 ce qu'il appelle une «sociocognitive approach of discourse» $(1985,1993,1997)$, qui s'inspire de la psychologie cognitive, repose sur la notion de «mental model » proposée par Johnson-Laird et s'appuie sur les références suivantes :

Cognitive mental model theory was first introduced by Johnson-Laird (1983) and van Dijk and Kintsch (1983). For recent collections of work on mental models, see Oakhill and Garnham (1996) and Van Oostendorp and Goldman (1998). For the 
obvious link of my theory with a cognitive theory of relevance, see Sperber and Wilson (1995) - (Van Dijk 2006 : 175).

10 Son appel aux sciences cognitives s'adresse donc à la psychologie cognitive, adresse naturelle pour un chercheur qui travaille au croisement des théories discours-texte et de la psychologie sociale, interface bien représentée et même disciplinarisée par la «critical discourse analysis $»^{4}$. Van Dijk définit le « mental model», permettant une « cognitive theory of discourse » comme

[...] a subjective representation of the events or situation that discourse is about. That is, understanding text or talk not only involves constructing a mental representation of its (intensional) 'meaning', but also and ultimately, a mental representation of its (extensional) 'referent' as the participants subjectively define it by constructing a mental model for it $(2006: 169)$.

Les modèles mentaux sont pour lui des interfaces articulant les relations entre textes et discours d'un côté, macrostructures sociétales de l'autre :

Thus social representations in our minds (such as socially shared knowledge, beliefs, attitudes and ideologies) are assumed to act as the necessary interface between micro-level interactions and individual text and talk, on the one hand, and societal macro-structures, on the other hand (1993: 107).

On constate que les modèles mentaux sont ici définis comme des représentations, croyances, attitudes, idéologies, etc., notions du répertoire de la psychologie sociale, sans dimension spécifiquement cognitive. Van Dijk ne propose pas, en effet, d'interrogation sur la nature de l'esprit, et ne prend pas position dans le débat entre internalisme et externalisme. Son emploi de cognition et cognitif semble donc au premier abord plus courant que spécialisé, et recouvrir davantage la question de la représentation que celle de la cognition. Son emploi de l'étiquette social cognition (1989) confirmerait cette hypothèse puisqu'il ne se réfère pas aux travaux qui, en sociologie et anthropologie cognitive, se réclament de ce courant, construit à partir des années 1990 contre le cognitivisme orthodoxe (voir infra 1.3.), mais au champ de la psychologie sociale qui sous cette dénomination propose l'étude des représentations et savoirs partagés.

Van Dijk précise à plusieurs reprises que les modèles mentaux ne sont pas observables, mais constituent une hypothèse explicative de certaines caractéristiques des textes et des discours :

In this paper we shall also ignore the specific representational format of models, but simply assume that they are networks that may be represented by propositions organized by schematic categories, like Participant or Setting. [...] Models simply explain many properties of text processing that were hitherto obscure or ignored, or dealt with in more ad hot ways (1997: 191).

Précisons que son dernier texte de 2006 ne précise pas non plus le format ou la nature de ces modèles mentaux, mais insiste plutôt sur leur non-observabilité :

As far as I know there is at present only one coherent, explicit and empirically warranted approach that meets these conditions: a sociocognitive account of context. In line with various earlier proposals, for instance in social psychology and sociology, I thus propose that contexts are not 'objective' or 'deterministic' constraints of society or culture at all, but subjective participant interpretations, constructions or definitions of such aspects of the social environment. From what we know about minds, such 'definitions' are mental, and in many situations they are only mental, and not expressed or formulated in discourse, although they may influence discourse (2006: 163). 
D'un point de vue linguistique, pertinent ici dans la mesure où il est question de productions textuelles et discursives, l'affirmation est un peu surprenante : on sait en effet que les travaux en linguistique cognitive, de la plus «orthodoxe» (paradigme computationnel) à la plus « californienne » (grammaires cognitives), de la plus syntaxique (grammaire chomskyenne) à la plus sémantique (courant fonctionnaliste), ont pour but, justement, de définir les cadres mentaux et de repérer les formes de langue qui en sont en même temps des productions et des configurations. Le choix de références en psychologie sociale ou cognitive entraîne donc, presque inévitablement, une version faible de la cognition, faible par rapport à l'objet choisi, qui est d'ordre langagier. Van Dijk précise d'ailleurs lui-même qu'il n'adopte pas les méthodes de la linguistique cognitive. D'où, à mon sens, une définition circulaire du contexte, qui ne permet pas vraiment la mise en œuvre d'un protocole d'observation de la matérialité textuelle ou discursive :

Unlike discourse or interaction, contexts are usually not 'observable' at all, whether traditionally defined as situational or societal constraints or as defined as mental constructs. (Incidentally, text and talk are only 'observable' in a very specific sense of 'being public', presupposing shared member's knowledge, because obviously the grammatical and other discursive structures, including those of meaning, cannot be directly 'seen', but are also results of the interpretations of participants.) Contexts only become 'observable' by their consequences on discourse, or vice versa, by the influence of discourse on social situations (2006:164).

Il est emblématique à cet égard que Van Dijk emploie désormais le mot cognition au pluriel (the cognitions), dans le sens de «shared knowledge », les savoirs partagés, donc au sens de contenus informationnels (2006).

On comprend que dans cette approche la cognition est une hypothèse explicative, assez puissante dans son principe, mais faible dans sa mise en œuvre et ses virtualités méthodologiques: sans observable, elle ne permet pas de description possible des processus cognitifs, des formes ou des contenus mentaux, et des formes langagières ou discursives qui leur sont interreliées. Un des auteurs du numéro de Discourse Studies sur discours et cognition formule d'ailleurs très joliment, et sans doute involontairement, le statut de la cognition dans cette approche : « How can we claim to be studying meaning, interpretation, understanding, and particularly those central concepts norms, premises, and cultural values, without venturing somehow into cognition ?» (Fitch 2006: 53). Venturing: la cognition semble en effet dans cette approche plus une terre d'aventure qu'un choix théorique.

\subsection{Une version plus robuste : la cognition externe}

18 L'articulation entre discours et cognition proposée ici est issue d'une réflexion théorique sur le discours informée par la sémantique cognitive américaine (Lakoff, Lakoff, Johnson) et par certaines approches françaises (par exemple celle de Nyckees 2003 et ici-même), ainsi que d'une interrogation sur les liens entre structures cognitives et productions discursives en termes de formes linguistiques et non en termes de contenus informatifs. Pour articuler l'approche cognitive aux dispositifs théoriques de l'analyse du discours, je remonterai au palier de la cognition non linguistique, suivant en cela les paradigmes qui inscrivent la cognition linguistique dans l'ensemble des activités humaines, selon une approche constructiviste (Fuchs $2004: 11$ et ss.). Il s'agira donc de passer par des versions philosophiques et socio-anthropologiques de la cognition, pour revenir ensuite sur le niveau linguistique. Je ne plaide pas l'inter- ou pluri- disciplinarité, mais l'appel à des 
disciplines ou domaines contributifs afin d'enrichir le répertoire théorique et méthodologique de la linguistique discursive.

La cognition sociale est constituée d'une myriade de travaux, discussions et orientations, qui proposent depuis les années 1990 une alternative à la cognition dite classique ou traditionnelle reposant sur l'hypothèse internaliste. Le paradigme internaliste s'est élaboré à partir des années 1950 autour de l'intelligence artificielle, de la psychologie cognitive, puis de la philosophie, de la linguistique et des neurosciences; le paradigme externaliste, ou social et culturel ${ }^{5}$, vient plutôt de la sociologie, de l'anthropologie, et dans une moindre mesure de la psychologie et de la philosophie. Les approches de la cognition sociale reposent sur des choix théoriques concernant la nature de l'esprit et la définition du contexte comme environnement matériel.

\subsubsection{L'extériorité de l'esprit}

On connaît le débat ancien sur la nature de l'esprit: soit l'on pose une frontière entre " skin and skull " et l'on considère que ce qui est en dehors du corps est en dehors de l'esprit; soit l'on considère que la production du sens et des connaissances se fait également dans l'environnement extérieur. J'accepte l'hypothèse, très anti-cartésienne (mais on sait depuis Damasio que même Descartes fait des " erreurs ») et apparemment contre-intuitive, surtout pour un esprit français de formation académique, de l'extériorité de l'esprit, et donc de la mémoire et de la cognition. Il y a cependant deux manières de penser cette extériorité : soit l'extérieur est un réservoir dans lequel l'esprit vient se ravitailler, soit l'environnement est actif dans la production du sens et des connaissances. Cette dernière position, qui est à la source de la notion de «technologie discursive " présentée en 3., est défendue par Clark et Chalmers dans un célèbre article " The extended mind». Il proposent un "active externalism», issu de la prise en compte d'interactions entre l'homme et les artefacts bien identifiées par les travaux en cognition sociale :

It is not just the presence of advanced external computing resources which raises the issue, but rather the general tendency of human reasoners to lean heavily on environmental supports. Thus consider the use of pen and paper to perform long multiplication (McClelland et al. 1986, Clark 1989), the use of physical rearrangements of letter tiles to prompt word recall in Scrabble (Kirsh 1995), the use of instruments such as the nautical slide rule (Hutchins 1995), and the general paraphernalia of language, books, diagrams, and culture. In all these cases the individual brain performs some operations, while others are delegated to manipulations of external media. Had our brains been different, this distribution of tasks would doubtless have varied (Clark, Chalmers $1998: 12)^{6}$.

21 L'hypothèse de l'«active externalism » a des conséquences épistémologiques importantes, que les auteurs signalent explicitement. C'est en effet une donnée importante de l'hypothèse de l'«extended mind» elle-même, et j'approuve cette intégration des conséquences épistémologiques d'une théorie à la théorie elle-même :

Thus, in seeing cognition as extended one is not merely making a terminological decision; it makes a significant difference to the methodology of scientific investigation. In effect, explanatory methods that might once have been thought appropriate only for the analysis of "inner" processes are now being adapted for the study of the outer, and there is promise that our understanding of cognition will become richer for it (Clark, Chalmers $1998: 8$ ). 
l'hypothèse de l' «active externalism" ne permet pas seulement d'enrichir la compréhension des mécanismes cognitifs; elle permet aussi de rendre compte de certaines actions sans passer par des dispositifs théoriques complexes qui appelleront le rasoir d'Occam :

By embracing an active externalism, we allow a more natural explanation of all sorts of actions. One can explain my choice of words in Scrabble, for example, as the outcome of an extended cognitive process involving the rearrangement of tiles on my tray. Of course, one could always try to explain my action in terms of internal processes and a long series of "inputs" and "actions", but this explanation would be needlessly complex. If an isomorphic process were going on in the head, we would feel no urge to characterize it in this cumbersome way. In a very real sense, the rearrangement of tiles on the tray is not part of action; it is part of thought (Clark, Chalmers $1998: 7$ ).

Certains chercheurs français, issus de la philosophie et de la sociologie, travaillent depuis une dizaine d'années à partir de cette hypothèse. Pour Quéré, l'extériorité de l'esprit est pensée de manière sociologique et il considère que les institutions et les pratiques sociales constituent un esprit externe :

Dans cette perspective, l'esprit est d'abord un "esprit objectif », incarné dans les institutions, les pratiques établies, les lois, les us et coutumes d'une société. La capacité de penser ou d'acquérir des connaissances est moins alors une capacité de former et de manipuler des représentations internes qu'une capacité de s'engager dans des pratiques sociales qui sont animées et articulées par des idées communes, des croyances légitimes et des systèmes conceptuels impersonnels (Quéré 1998: 161).

Cette idée est également défendue dans le domaine des sciences du langage par Auroux qui pose l'existence de « structures cognitives externes », dans le cadre de sa réflexion sur les technologies intellectuelles et les technologies de la langue :

L'hypothèse contraire [à la cognition dans la tête] repose sur l'existence de structures cognitives externes à l'individu. Ces structures ont connu leur développement grâce à la technologie intellectuelle de l'écriture; elles dépendent également d'instruments externes (livres, bibliothèques, instruments de calcul et d'observation, etc.), ainsi que de structures sociales de productions et de cumulation de connaissances (encyclopédies, sociétés savantes, réseaux culturels de production et de reproduction du savoir). Le processus cognitif dépend de la structuration sociale tout comme en dépend la production des richesses (Auroux $1998: 6)$.

Il faudrait également citer Conein, Lahlou, Thévenot pour la sociologie cognitive ${ }^{7}$ et l'ergonomie, Latour et la sociologie associationniste, mentionner le paradigme énactiviste qui se développe actuellement à partir des travaux de Varela, parler également des recherches en sémiotique qui ont depuis longtemps intégré l'articulation entre la pensée et l'environnement extérieur, ainsi que de la médiologie, et d'autres travaux encore (pour une revue du débat externalisme vs internalisme voir Brassac (dir.) 2006). Je remarque que les sciences du langage, en particulier dans leur composante texte-discours, sont assez loin de ce type d'interrogation et maintiennent la distinction interne vs externe sous la forme linguistique vs extralinguistique. Mais cette opposition binaire ne permet pas selon moi de rendre compte d'un nombre assez important de phénomènes langagiers, comme la forme morphographique de la liste, certaines formes conversationnelles directement appuyées sur des artefacts ou des objets culturels, des formes rhématiques (qui ont leur thème dans l'environnement extérieur) ou des genres discursifs comme l'éloge qui s'articulent avec une composante rituelle matérielle (cérémonie et objets, voir 
par exemple Paveau 2006b). Je souscris donc pour ma part à l'hypothèse de l'«active externalism ", tout en restant sur une position constructiviste qui m'évite la soumission à la pensée binaire : je préfère poser un continuum entre les cadres internes et les manifestations externes de l'esprit et je préciserai plus loin que les prédiscours se logent autant « dans la tête » que dans les environnements extérieurs humains et non humains.

\subsubsection{Repenser le contexte}

L'appel à la philosophie de l'esprit et à la cognition sociale permet donc de repenser de manière dynamique la notion de contexte. $\mathrm{Si}$, en effet, l'on admet l'hypothèse de l'extériorité de l'esprit, et donc de la faculté de langage et, par conséquent de la compétence discursive, alors des horizons s'ouvrent: ce que l'on appelait dans la tradition de l'école dite française d'analyse du discours ${ }^{8}$, les « conditions socio-historiques de production ", dans une perspective marxiste qui fait la part belle à la praxis, ces conditions peuvent être étendues aux données environnementales. Le destin de la notion de praxis est d'ailleurs particulièrement emblématique de la propension cartésienne française à maintenir la distinction «skull and skin»: chez les premiers analystes du discours inspirés par le matérialisme historique interprété par Althusser, la praxis est volontiers définie en termes de matérialité :

Nous dirons donc, à ne considérer qu'un sujet, (tel individu) que l'existence des idées de sa croyance est matérielle, en ce que ses idées sont ses actes matériels insérés dans des pratiques matérielles, réglées par des rituels matériels eux-mêmes définis par l'appareil idéologique matériel dont relèvent les idées de ce sujet (Althusser 1995 [1970]: 301 ; ital. de l'auteur)

Si les "matérialités discursives» de l'école française (Conein et al. (dir.) 1981) ne désignent pas directement l'environnement concret de notre existence mais, indirectement, la dimension socio-idéologique des discours, il n'en reste pas moins que ce sont bien les pratiques matérielles des locuteurs inscrits et même englués dans la société et ses appareils idéologiques qui sont en cause. Quand, par exemple, le courant de la linguistique praxématique revendique la prise en compte des "expériences pratiques d'appréhension et de transformation du monde dans sa matérialité", souligne l'importance des "intérêts matériels et symboliques", et critique "des théorisations régies par un processus d'abstraction idéaliste [qui] ont cru pouvoir en [la praxis] faire l'économie » (Détrie et al., 2001 : 268), on s'attend donc à ce que la notion de contexte soit informée par les environnements matériels. Mais l'article contexte du même ouvrage ouvre timidement sur le domaine de l'action et se concentre sur les «comportements corporels » et la notion de situation telle qu'elle est travaillée en ethnographie de la communication et en analyse conversationnelle, autrement dit n'envisage le contexte environnemental matériel que dans le cadre de la relation interindividuelle, comme un arrière-plan ou une ressource (Détrie et al. 2001: 62-63). On comprend donc que les tenants de la praxis n'envisagent pas une articulation directe entre discours et pratique matérielle : la praxis est, comme le souligne très justement Guilhaumou, encore pensée comme une rupture ${ }^{9}$, etsemble impensée et peut-être même impensable comme articulation ou continuum entre discours et réalité.

C'est ce continuum que je propose ici, en m'appuyant sur l'hypothèse de l'« extended mind " et sur les travaux en cognition sociale distribuée (j’y reviens en détail en 3.): le contexte intègre les données environnementales qui se constituent à la fois de cadres internes (les prédiscours comme cadres de savoirs, de croyances et de pratiques) eux- 
mêmes informés par les données externes, et des réalités extérieures de notre environnement matériel concret (décors naturels ou artificiels, espaces, objets culturels et techniques, artefacts, supports, etc.). Dans l'exergue de Daninos qui figure au début de cet article, la nappe et "ce rond stupide que dix fauteuils délimitent» sont aussi importants que les petites boîtes cognitives subtilement énumérées par le penseur mondain.

Cette conception implique une sorte de réversibilité subjective du contexte: penser l'esprit, la mémoire et la faculté de langage comme externes implique par retour de (re)penser le contexte comme interne à la conscience et à la mémoire. Et cette conséquence en implique deux autres: poser comme terrain d'observation pour la linguistique discursive la continuité entre ces deux sources cognitives, et sortir de la relation interindividuelle pour examiner les relations entre les agents humains et les agents non humains, les deux étant considérés comme des agents psychiques. L'anecdote racontée par Duranti constitue une sorte d'illustration empirique de ces implications :

When my son Marco was five and a half I took him to his first piano lesson. I sat on a couch while the young teacher started to explain the arrangement of the keys. 'You see,' he said, 'there are three black keys here and then two black keys over here and then another three and two, and three and two.' Marco turned away from the keyboard to look up at the teacher and, in a very matter-of-fact tone, said, 'It's a pattern.' The teacher smiled, nodded, and moved right away to the next item on his agenda, 'Mary Had A Little Lamb', a classic first song for beginning piano players in the US (Duranti 2005 : 409).

Il en tire une sorte d'impératif catégorique pour l'étude scientifique de la cognition humaine : "More generally, any time we examine interactions that make use of cultural artifacts, including new technologies [...], we are forced to rethink communication as something more than an exchange of thoughts from one individual (or group) to another » (Duranti $2005:$ 420).

Cette nouvelle version du contexte amène une autre question: peut-on soutenir sérieusement que le contexte "pense », qu'il est « intelligent», autrement dit, que «le cockpit se souvient réellement de ses vitesses ", selon le titre célèbre de l'article de Hutchins (1994 [1991]), ou la formule n'est-elle que métaphorique, les agents humains «faisant parler », pour ainsi dire, le contexte matériel et ses réalités non humaines? Cette discussion est présente dans les travaux en cognition sociale et sociologie des sciences. D'un côté des chercheurs qui, à la suite de Latour et de ses propositions pour une « anthropologie symétrique » (c'est-à-dire qui examine à la fois les sujets humains et les objets non humains dans leurs assemblages et leurs interactions), défendent le rôle cognitif des réalités non humaines: «Les sociologues ne chercheraient-ils pas midi à quatorze heures en construisant le social avec du social ou en maçonnant ses fissures avec du symbolique, alors que les objets sont omniprésents dans toutes les situations dont ils cherchent le sens? En leurs mains, la sociologie ne reste-t-elle pas sans objet?», se demande Latour dans un article sur "l'interobjectivité » (1994:592). Son hypothèse déplace les enjeux épistémologiques et théoriques des sciences sociales et sa description du rôle des objets dans la vie humaine est particulièrement intéressante pour penser, dans le champ de la linguistique, une articulation entre agents humains et non humains :

Things (or quasi-objects or risk, the word does not matter) have the peculiar feature of not being divisible into primary and secondary qualities. They are much too real to be representations, and much too disputed, uncertain, collective, variegated, divisive to play the role of a stable, obdurate, boring primary qualities, furnishing the universe once and for all. What the social sciences, together with the 
natural, can do, is to represent those things in all of their consequences and uncertainties to the people themselves (1999: 107).

\section{l'environnement non humain. Giere et Moffatt pensent par exemple que ce n'est pas} l'environnement qui « sait », mais les humains grâce à l'environnement :

Accepting the idea that cognition may be distributed throughout a system comprising both humans and artifacts may tempt one to ascribe cognitive agency to the larger system, to say it is the system that knows, perhaps even consciously knows [...]. We think this temptation should be resisted. It is possible to think of the whole system as producing knowledge while maintaining that it is only the humans who come to know the results, in some completely ordinary sense of knowing (Giere, Moffatt 2003 : 304).

Quelle que soit la position que l'on adopte sur la nature exacte de la source cognitive gisant dans l'environnement non humain, il me semble qu'il y a dans la cognition sociale des propositions aptes à enrichir les approches texte-discours du contexte. Je ne souhaite pas naturaliser à l'extrême la cognition et renoncer aux cadres internes ; mais l'idée d'une cognition externe me semble particulièrement importante pour l'étude des productions discursives en contexte, c'est-à-dire in vivo. Je présente maintenant la notion de prédiscours élaborée dans Paveau 2006a, un peu infléchie depuis vers la cognition distribuée via une réflexion sur le rôle des objets dans la cognition linguistique (Paveau, Rosier 2005b, 2007).

\section{Le concept de prédiscours}

Une fois admise l'hypothèse d'une construction des perceptions et connaissances qui passe par les agents humains mais aussi par les relations qu'ils entretiennent avec le monde non humain, reste à saisir et décrire les processus par lesquels se fait cette construction. On a souvent parlé de «boîte noire» (Kerbrat-Orecchioni 1986) ou de «boîte de Pandore» (Latour 2001) pour décrire les données encyclopédiques et environnementales que les individus mobilisent pour la production des discours. Cette image est sans doute plus sociale et institutionnelle que scientifique, des disciplines constituées sur la boîte fermée craignant légitimement son ouverture. En effet, si la linguistique doit faire du linguistique avec du linguistique, et la sociologie du social avec $\mathrm{du}$ social, alors la prise en compte de l'extralinguistique et de l'objectal modifierait l'épistémologie de ces disciplines. La linguistique pense en effet l'environnement, surtout quand il est naturel ou objectal, en dehors d'elle. Même les courants les plus bienveillants envers la prise en compte de l'extralinguistique continuent à le penser comme du nonlinguistique. Je préfère pour ma part penser le rapport entre linguistique et extralinguistique sous la forme d'un continuum, et ne pas faire comme si les humains pensaient et parlaient dans une fière autonomie par rapport au monde qui les entoure. La notion de prédiscours est une proposition pour décrire ce continuum.

\subsection{Des cadres prédiscursifs collectifs}

Dans cette perspective, j'appelle données prélangagières, à la suite de Nyckees 1998 par exemple (qui parle de "catégories prélinguistiques») un ensemble de perceptions et de représentations issues de l'environnement, de la culture, de la mémoire ou des interactions, qui sont préorganisées par la dimension linguistique et élaborées et 
reconfigurées en discours. Prélangagier n'est pas pris au sens de la psycholinguistique (par exemple "enfant prélangagier ", i.e. à un stade avant l'acquisition du langage) mais se réfère, dans une réflexion qui souhaite articuler le sémantique, le discursif et le cognitif, à la manière dont les sujets construisent le sens en discours à partir de données qui ne sont pas seulement internes mais qui peuvent résider dans les contextes externes.

Comme mon domaine de travail est le discours, je parle, pour rendre compte de ces données antérieures, de prédiscours, définis comme un ensemble de cadres prédiscursifs collectifs (savoirs, croyances, pratiques) qui ont un rôle instructionnel pour la production et l'interprétation du sens en discours. Les prédiscours sont des opérateurs dans la négociation de la propagation et de la distribution du sens dans les collectivités humaines, des plus restreintes et informelles aux plus larges et instituées (je fais mienne la définition que Gilbert 2003 donne des "sujets pluriels $»^{10}$ ). Je considère, dans la perspective de la cognition sociale, les prédiscours comme des données propagées par une mémoire que j'appelle cognitivo-discursive, résultat d'un retravail du concept de " mémoire discursive » (Courtine 1981) et de "mémoire interdiscursive " (Moirand 2003, 2007 et ici-même), et distribuées par un certain nombre d'agents internes et externes comme les artefacts ou les technologies cognitives. Je dis distribuées, car je pense que la notion de partage, souvent alléguée quand il s'agit de rendre compte du fait que les individus possèdent une culture commune (les fameux « savoirs partagés »), est trop peu dynamique. Je ferai donc appel (voir infra 3.) aux acquis de la cognition distribuée (Havelange 2001, Hutchins 1995, Conein 2005) pour montrer plus précisément que les prédiscours sont le résultat d'une cognition humaine collective qui reçoit la contribution d'outils cognitifs externes.

Les prédiscours ne sont pas des segments discursifs identifiables (des discours qui auraient été produits avant, ce qui les tireraient par exemple vers le discours rapporté) mais des cadres préalables tacites, signalés dans les discours par un certain nombre de phénomènes langagiers. J'appelle tacites des éléments qui ne sont pas susceptibles de recevoir une explicitation (ce qui les distingue des cadres implicites), mais qui ont un fort pouvoir instructionnel. Les prédiscours sont dotés de six caractéristiques qui les rendent linguistiquement et cognitivement analysables :

- leur collectivité, résultat d'une co-élaboration entre les individus et entre l'individu et la société ; je ne réduis pas la collectivité des cadres préalables aux échanges entre les locuteurs, mais je les élargis aux interactions entre les individus, leurs institutions et les environnements naturels et artificiels dans lesquels ils sont intégrés. Les prédiscours informant par exemple un échange entre un enseignant et ses élèves dépendent tout autant des arrière-plans culturels des participants que des meubles, artefacts et objets divers présents dans l'environnement de la classe.

- leur immatérialité, la prédiscursivité étant d'ordre tacite ; l'étymologisme par exemple, qui consiste à fonder une argumentation sur le recours à l'autorité d'un sens supposé pur et vrai des mots, constitue un appel au prédiscours de la loi de la langue en même temps qu'à celui, artefactuel, du dictionnaire étymologique ou de toute autre liste ressortissant à une technologie discursive. 
- leur transmissibilité, sur l'axe horizontal de communicabilité encyclopédique (l'interrogation générique du type «X n'est-il pas ? » constitue par exemple un appel au prédiscours de la doxa impliquée par la forme elle-même) et l'axe vertical de la transmission via les lignées discursives (le rôle de la mémoire, que je détaillerai plus bas en examinant le sémantisme souple du nom propre en discours).

- leur expérientialité, puisqu'ils permettent au sujet d'organiser mais aussi d'anticiper son comportement discursif. Toutes les typologies de la psychologie populaire (des signes du zodiaque aux types d'acheteur ou d'amoureux que nous sommes d'après les " tests psycho " des magazines) me permettent, comme locutrice, de me diriger dans l'ensemble des discours et des comportements sociaux.

- leur intersubjectivité, les critères de mobilisation étant véri-relationnels et non logiques. Cela veut dire que les prédiscours ne sont pas mobilisés parce qu'ils sont vrais vs faux, mais parce qu'ils conviennent à l'ensemble des agents, qu'ils sont reconnus comme opératoires et approuvés dans le cadre des relations intersubjectives. Que « les femmes pleurent » et que « les hommes mentent », comme l'indiquent les titres des best-sellers des Pease ${ }^{11}$, constitue par exemple des vérités relatives et intersubjectives qui recourent à des prédiscours tacites sur la différence des sexes.

- leur discursivité enfin, puisqu'ils sont langagièrement signalés. Lexicologisme, lexicographisme, noms propres, certaines formes de deixis, typologie, métaphore, antithèse : autant de formes d'appel à des prédiscours distribués dans les esprits et les environnements culturels et matériels de locuteurs (pour les analyse de détail à partir de ces six caractéristiques voir Paveau 2006a).

Je choisis ici de commenter en détail l'une de ces caractéristiques, la collectivité, avant de présenter la notion de technologie discursive.

\subsection{Du partage à la distribution : une version dynamique de la collectivité}

La question de la collectivité est cruciale actuellement en sciences du langage et dans l'ensemble des sciences humaines: les résultats des travaux en sciences cognitives, les avancées en sociologie comme les évolutions en particulier technologiques des communications rendent désormais quasiment impossible de penser l'individu dans son autonomie métaphysique traditionnelle. Mais tout rabattre sur la notion de " collectif », sans autre forme d'examen ne permet pas de repenser la nature des groupes et des associations humaines.

\subsubsection{L'individu collectif}

La collectivité des prédiscours fait donc émerger la vieille question du rapport entre individuel et collectif, apparentée à la distinction interne / externe décrite plus haut. Il me semble que la pertinence de cette antithèse exclusive est sérieusement remise en 
cause désormais en sciences humaines et sociales, et qu'il est plus efficace, et surtout plus exact, de penser un continuum entre les deux. La psychologie sociale ${ }^{12}$, les sciences sociales dans leur version marxiste ${ }^{13}$, et l'ensemble des travaux issus des approches sociales de la linguistique (sociolinguistique, linguistique sociale, et, dans une certaine mesure, analyse du discours) ont certes légitimement mis l'accent sur les déterminations collectives de nos manières d'être et de dire dans les soixante dernières années. On y a cependant toujours conservé le mythe de l'individu autonome, ce que Flahault appelle le paradoxe de Robinson, remarquant que Marx lui-même est persuadé que l'individu précède la société (Flahault 2005). Ce que montrent à la fois les sciences cognitives internalistes (les neurosciences en particulier) et les versions sociales et culturelles de la cognition, c'est que l'individu, dont l'existence et les manifestations en tant que telles ne sont pas pour autant contestées, ne pourrait cependant pas exister sans ses contextes extérieurs, qu'ils soient culturels, objectaux, naturels, sociaux, etc. Pour aller vite, Robinson n'existe pas sans Vendredi, ou, plus exactement sans au moins Vendredi. Mais, sans doute parce que ce déplacement en implique d'autres, et pas des moindres (repenser les oppositions subjectif $v s$ objectif, singulier vs pluriel, interne $v s$ externe), « l'inconscient culturel » français ${ }^{14}$ et en particulier celui de la linguistique, a du mal à se défaire de ces prédiscours scientifiques de l'opposition voire de la distribution complémentaire. Il me semble cependant préférable de penser les rapports entre individuel et collectif de manière scalaire, quitte à se heurter à des difficultés d'analyse et d'interprétation faute de cadres de travail adéquats, qui doivent alors être inventés.

41 Je pars donc de l'idée qu'il existe une co-élaboration à la fois entre les productions discursivo-cognitives individuelles de chacun (mes cadres préalables et ma compétence discursive ne peuvent s'activer qu'en collaboration avec les tiens) et entre les productions individuelles et sociales (mon comportement individuel est imprégné de social et corrélativement les phénomènes collectifs d'une société sont marqués par les innovations individuelles). Le problème est alors d'identifier et de décrire les points de passage des cadres prédiscursifs entre les individus, et entre l'individu et la société, considérée ici dans sa dimension globale (culture, institutions, artefacts, objets, décors, etc.). Dans les travaux actuels au sein des disciplines qui ont à faire avec le discours, circulent deux concepts-étiquettes qui proposent une forme de solution à ce problème: le partage et l'intersubjectivité. Je dis "une forme de solution », car il me semble que les procédures par lesquelles sont réalisées les articulations décrites plus haut ne sont pas réellement décrites et expliquées.

\subsubsection{La mémoire du cockpit}

Le partage apparaît par exemple sous l'étiquette de «savoirs partagés» ou " connaissances partagées ", étiquette qui rejoint partiellement celle d'implicite, comme le montre l'historiographie de cette notion établie par Kerbrat-Orecchioni $(1980,1986)$. La notion est également convoquée en sémantique, dans le traitement des stéréotypes par exemple (par exemple dans les travaux Kleiber sur les stéréotypes du type «j'ai acheté un stylo et la plume est tordue ", " on est arrivé dans un village et on a cherché l'église ", où l'anaphore est justifiée par des "connaissances partagées»). Mais on en reste à une explication, voire à un slogan, et le mécanisme du partage n'est pas décrit. Les hypothèses de la cognition sociale se sont d'ailleurs également déplacées du partage à la distribution, considérée comme plus dynamique. Le courant de la cognition partagée, représenté par exemple par Roschelle, insiste sur l'aspect social de la cognition, mais ne prend cependant 
pas assez en compte l'ensemble de l'environnement non humain: "[...] collaborative problem solving takes place in a negotiated and shared conceptual space, constructed through the external mediational framework of shared language, situation and activity not merely inside the cognitive contents of each individual's head " (Roschelle, Teasley, 1995 : 71). Langage, situation et activité : on est dans une définition du contexte proche de l'interactionnisme, mais la dimension active des autres éléments de l'environnement manque.

La notion philosophique d'intersubjectivité, plus forte également que celle de partage, proposée par Husserl dans les années 1910 et devenue très courante en philosophie à partir des années 1940, est volontiers mobilisée par les linguistes dans le cadre d'une interrogation sur la collectivité du sens ou des représentations : Benveniste en use dès les années 1950, Culioli vers les années 1965-1975, avant que n'apparaisse dans son travail la notion de co-énonciation; Kleiber de son côté parle de «stabilité intersubjective » dans les années 1990 pour définir le sens. Le postulat de l'intersubjectivité est en effet nécessaire pour rendre compte du fait que les prédiscours, savoirs, croyances et pratiques préalables et collectifs de tous les locuteurs d'un groupe ou d'une communauté, font l'objet d'un accord interindividuel, accord qui constitue de ce fait une forme de collectif. Mais parler d'intersubjectivité n'est pas suffisant: cela ne nous dit pas comment les prédiscours se diffusent concrètement entre les différents agents humains et non humains (d'où la nécessité de faire également intervenir l'«interobjectivité » que mentionne Latour).

Dans l'approche de la cognition distribuée, on prend en compte ces éléments objectaux et techniques dans le cadre d'une observation directe. En effet, si l'on reprend le cas princeps de l'activité de pilotage dans un avion (Hutchins 1994 [1991]), on constate que l'ensemble des représentations présentes dans le système choisi (états des indicateurs, interactions entre individus, instruments, blocs-notes, etc.) a fait l'objet d'une observation et d'une évaluation. Dans le cadre d'une analyse cognitive ou discursive traditionnelle, le niveau d'analyse aurait été celui des interactions humaines. Cela veut dire que c'est la nature de l'unité d'analyse qui se modifie : l'unité pertinent devient le cockpit tout entier. L'accent se porte donc sur l'analyse des propriétés cognitives du cockpit (vu sous l'angle d'un système cognitif) et non pas sur l'analyse des propriétés cognitives des individus.

Je voudrais montrer maintenant plus précisément pourquoi l'hypothèse de la « distributed cognition » me semble la plus pertinente pour le traitement véritablement contextualisé des productions discursives.

\section{La technologie discursive : le discours et ses outils}

De même qu'il existe des cognitive tools, notion proposée par Resnick pour décrire des instruments qui permettent de penser et de catégoriser, matériels (une base de données ou un agenda) ou non (pour Vygotski par exemple, le premier outil est le langage luimême), il existe à mon sens des discursive tools ou outils discursifs qui contribuent à la construction des discours. 


\subsection{La notion de technologie discursive}

47 L'idée d'une technologie cognitive n'est pas nouvelle et trouve son origine, en France, dans les travaux de Leroi-Gourhan pour lequel les dispositifs techniques constituent une forme d'extériorisation des organes sensori-moteurs, mais qui contribuent de manière interne à la cognition. Il ne s'agit pas d'instruments qui seraient simplement des moyens, mais de véritables paramètres de la cognition. Selon Havelange par exemple, les artefacts techniques médiatisent la boucle sensori-motrice et s'avèrent constitutifs de la cognition anthropologique et de la mémoire sociale :

Loin de pouvoir être réduite à une série d'objets circonscrits dans un espace et un temps positifs, la technique présente ainsi une dimension transcendantale qui dépasse de très loin le schème instrumental de moyens assujettis à des fins prédéterminées: elle est constitutive de l'hominisation, en tant que genèse de la subjectivité, de la socialité et de l'historicité (Havelange 2001 : 34).

À partir de ces données, je propose la notion de technologie discursive pour désigner des techniques, artefacts ou objets qui configurent, formatent, et informent les prédiscours en vue de l'élaboration des discours. Il peut s'agir d'outils linguistiques (listes lexicales, grammaires, manuels, dictionnaires, mémentos, listes, guides de conversation, etc.), ou plus largement discursifs, des ex-voto aux emballages alimentaires, en passant par les faire-part de deuil et les tatouages, et de nombreux autres artefacts comme les blocsnotes ou les logiciels, les carnets d'adresse, les agendas, les calendriers, le tout sous forme papier ou électronique. Il peut s'agir aussi, comme le signale Conein insistant sur la dimension collective du système cognitif, de «données publiques, méthodes instrumentées, comme tous les artefacts permettant d'acquérir, de stocker, de visualiser une quantité d'informations qu'un seul individu ne peut traiter » $(2005: 176)$.

La notion de technologie discursive s'inspire de celle de «technologie intellectuelle », que Bourdieu et Passeron proposaient dès 1970. Ils se situaient dans le cadre d'un militantisme sociologique prenant appui sur l'étude des pratiques de classes sociales, et leur propos est parfaitement valable pour la question qui m'occupe: comme la technologie intellectuelle, la technologie discursive semble réduite à un outil d'acculturation ou d'apprentissage, alors qu'elle est partie intégrante de l'usage de la langue et de la production discursive. Une publication récente sur l'œuvre de Goody a fait émerger la notion de "technologie de l'intellect ", que l'anthropologue proposait dès... 1968 et qui semble avoir eu une existence bien souterraine jusqu'à présent (Kara, Privat (dir.) 2006).

L'idée d'une contribution des outils, objets et artefacts à la production des discours n'est donc pas nouvelle ${ }^{15}$ mais reste en effet circonscrite jusqu'à présent à des activités particulières comme l'apprentissage ou le travail.

51 En France, les travaux des membres du réseau «Langage et travail » créé en 1986 constituent désormais une référence en matière de réflexion pluridisciplinaire (linguistique, sociologie, ergonomie, anthropologie, économie) sur la cognition au travail. L'accent est particulièrement mis sur les « matérialités » qui contribuent à l'action et à la parole :

Les différents univers sémiotiques auxquels l'automation confronte les salariés - la langue courante orale, la langue écrite, les chiffres, les icônes, les maquettes possèdent des matérialités propres et partiellement distinctes auxquelles, par exemple, l'ergonomie est amenée à s'intéresser (Boutet 2001 : 104). 

vers une théorie générale de l'interaction entre agents humains et non humains dans la production des discours, qu'ils soient écrits ou oraux. De plus certains des travaux de ce courant maintiennent la distinction entre des activités de travail presque exclusivement langagières et d'autres fortement inscrites dans des milieux techniques. C'est à mon sens reconduire au sein même de l'articulation entre humain et non humain, la vieille distinction «skin and skull»: il y aurait quelque chose comme du langage fait (presque) uniquement avec du langage, ce qui constitue un obstacle pour une approche de l'ensemble de l'activité de langage articulant cadres internes et réalités externes humaines et non humaines.

L'approche de la cognition distribuée, elle aussi élaborée à partir de situations de travail et d'apprentissage, s'est en revanche généralisée à la cognition humaine dans son ensemble et des synthèses puissantes sont disponibles depuis les années 1990.

\subsection{Les acquis de la cognition distribuée}

54 J'ai décrit plus haut le champ de la cognition sociale qui propose une alternative à la cognition internaliste classique. Dans ce large domaine alternatif, plusieurs courants se sont constitués, dont celui de la cognition distribuée (distributed cognition). Le principe de base est celui de l'extended mind et les processus cognitifs sont décrits comme «distribués » entre les agents et leur environnement, celui-ci comprenant à la fois les objets et artefacts mais aussi les structures sociales. Le système cognitif n'est donc plus l'individu mais cet ensemble d'agents humains, non humains et sociaux, et l'unité d'observation et d'analyse est donc fortement modifiée par rapport à la cognition individuelle (pour une synthèse voir Conein 2005). Ce sont les travaux d'Hutchins et de ses collègues de San Diego qui ont ouvert ce domaine, dans lequel on peut compter des chercheurs comme Latour, Agre et Norman, ainsi que Conein, Quéré, ou Thévenot dans une version française plus philosophique de la sociologie cognitive. L'environnement n'est plus considéré comme un arrière-plan pour la cognition, mais comme un ensemble de «ressources environnementales». Les objets inanimés et les «données publiques»(Conein) ne sont plus des «aides périphériques» mais des formes de représentation externe qui interviendront dans les élaborations cognitives.

Afin de proposer un cadre d'observation et d'analyse adapté à la cognition linguistique et discursive, j'intègre aux principes et méthodes de la cognition distribuée deux modèles de rapport entre l'individu et les objets et artefacts, permettant de penser la disponibilité intentionnelle de l'objet.

Le modèle des affordances proposé par Gibson dès 1966 (pour le terme) puis développé dans les années 1970, et repris par Norman dans les années 1980, est bien connu. Une affordance est pour Gibson une propriété d'un objet ou un trait de l'environnement immédiat qui indique quelle relation on doit instaurer avec l'objet, en particulier quel usage on doit en faire. Les affordances sont des "action possibilities" latentes dans l'environnement, d'abord indépendantes des habiletés des agents puis, dans un second temps de la théorie, pertinentes aux agents (un escalier aux marches hautes n'afforde pas la même possibilité pour un très jeune enfant et un adulte). Norman retravaille cette théorie en envisageant les " perceived affordances », des affordances perçues par les agents et donc subjectives, ce qui fait des affordances de Gibson des affordances objectives (real pour Norman). Norman explique que dans une pièce comprenant un sofa et une balle de 
softball l'agent peut s'asseoir sur la balle et lancer le sofa parce que c'est objectivement possible. Mais il formule surtout une théorie générale de l'objet comme représentation externe qui permet de repenser efficacement le contexte de la production symbolique humaine :

Le besoin de naviguer et de vivre dans le monde requiert aussi que nous sachions nous rappeler les positions et les objets du monde, et utiliser ces objets comme rappel de séquences d'action. En ce sens, les objets du monde jouent un rôle de signe, et il n'est pas nécessaire d'amplifier cette aptitude naturelle pour qu'ils puissent servir de représentations, de symboles. Tout ce qu'il faut c'est un mécanisme associatif pour établir une correspondance entre objets, localisations dans l'environnement et pensées internes. Une fois que ceci a été accompli, les objets du monde servent de système de symboles physiques, et le monde même est utilisable comme une banque de données efficace (Norman $1993: 17$ ).

En d'autres termes, les acquis de la cognition distribuée permettent de penser l'intentionnalité des objets, en tout cas ce que Tomasello appelle leur «disponibilité intentionnelle »: «[...] des objets ou des artefacts possèdent, outre leur disponibilité sensori-motrice, ce que nous pourrions appeler une disponibilité intentionnelle: il comprend les relations intentionnelles que l'autre entretient avec l'objet ou l'artefact et du même coup les relations intentionnelles qu'il entretient avec le monde par le biais de l'artefact » (Tomasello 2004 : 83). Cette intentionnalité de l'objet est évidemment le point fort de la théorie des affordances, et se retrouve sous une autre forme, l'attraction, dans le modèle français des attracteurs cognitifs de Lahlou, construit à partir d'une recherche sur le travail de bureau :

Appelons attracteur cognitif un ensemble d'éléments matériels et immatériels participant à une activité particulière, ensemble d'éléments qui se présentent simultanément à la perception du sujet. Nous posons que la perception conjointe de plusieurs éléments d'une même activité " attire » le sujet vers l'activité en question. Autrement dit : quand le sujet est mis en présence d'une bonne partie de la panoplie d'une activité donnée, il est « en condition » d'accomplir cette activité, et il a tendance « à s'y mettre » (Lahlou $2000: 89$ ).

L'attracteur, "invariant pragmatique " a un effet sur l'acteur, que Lahlou nomme « artisan cognitif ambulant» car les «data ( (données) s'articulent avec les «lata » (ce que les acteurs portent comme représentations). Trois facteurs sont déterminants pour évaluer la force des attracteurs: la prégnance (capacité à s'imposer au sujet), le coût (efforts du sujet pour accomplir l'activité) et la valeur (gain ou «non-perte » au cours de l'activité).

\subsection{Outils linguistiques et discursifs : des contributeurs cognitifs au discours}

Il ne me semble pas (ou plus, ce qui encore une autre question) possible, comme les développements précédents sur le contexte, l'extended mind et la cognition distribuée l'ont montré je l'espère, de "faire du linguistique avec du linguistique», pour paraphraser Latour. Il me semble nécessaire d'intégrer à la cognition linguistique cet environnement non humain, non réduit à la situation, au cadre social ou à la disposition des individus, qui nous est si évidemment familier et consubstantiel dans toutes nos activités, et pourtant si coutumièrement et légitimement absent des réflexions sur le fonctionnement du langage. Je pense comme Thévenot " que les choses sont personnalisées ou que la personne est consolidée et chosifiée par son entourage » (1998: 
136). Je propose pour le moment une première typologie des objets et artefacts qui contribuent à la construction du discours, et une description programmatique des modes de contribution en question.

On peut d'abord distinguer les outils et les objets, les premiers étant dotés d'affordances objectives explicites pour contribuer à former le discours, les seconds possédant des affordances subjectives et implicites.

Les outils sont linguistiques ou discursifs. Les outils linguistiques sont des objets déjà observés dans certains secteurs des sciences du langage (lexicographie, lexicologie, histoire des idées linguistiques), mais pour leur contenu et non pour leur statut d'objets. Ils sont faits pour enregistrer les compétences langagières : ce sont les dictionnaires, grammaires, listes lexicales, lexiques, jeux d'apprentissage, guides de correspondance ou de protocole, manuels de savoir-vivre, etc., présents à titre de traces ou d'instruments dans la production des discours. Ils constituent des représentations externes de la norme langagière et discursive et circulent comme tels dans le système cognitif composé des agents humains, non humains et des paramètres situationnels au sens large. Les outils discursifs sont des objets pour soutenir les compétences discursives et étayer les productions discursives, les affordances explicites suggèrent des usages langagiers et non des normes linguistiques comme les précédents: tableaux, petits papiers, brouillons, check-lists, «strips ", power point, post-it sont autant d'outils discursifs qui soutiennent cognitivement la production discursive. Les outils linguistiques et discursifs sont des artefacts, au sens que Norman donne à ce mot : «Les artefacts cognitifs acquièrent leur fonction en tant qu'outils représentationnels. En effet, je définis un artefact cognitif comme un instrument artificiel conçu pour conserver, rendre manifeste de l'information ou opérer sur elle, de façon à servir une fonction représentationnelle » (Norman 1993 : 28).

62 Les objets discursifs, dont les affordances pour le discours sont implicites et subjectives, ne sont pas conçus intentionnellement pour la production du discours. Les objets discursifs graphiques portent du langagier et sont dotés d'affordances discursives graphiques : monuments aux morts, emballages alimentaires, bouteilles, porte-clefs, linge brodé, marquoirs, objets gravés de toutes sortes (timbales, bagues, alliances), tatouages, etc. Les objets discursifs non graphiques ne portent pas de langagier, leurs affordances sont discursives non graphiques : ce sont des verres, briquets, foulards, cailloux, et les milliers d'objets qui font le quotidien de notre socialité.

Reste à se demander évidemment comment les représentations se propagent dans le système. Une réponse que ne renierait pas Daninos est celle que fournit le journal The Lancet de décembre 1953 : « Les grandes maisons de stylos ne sortant depuis vingt ans que des articles à corps trop mince et à plume trop dure, seraient responsables d'une part de la fréquence croissante de la crampe de l'écrivain, d'autre part, en littérature, de l'abondance anormale de récits ou essais étriqués et secs». Plus sérieusement, ma réponse est la notion de contribution, entre attraction et distribution. Les objets sont en effet des contributeurs cognitifs à la production du discours, qui donnent des instructions, de la même manière que les prédiscours, pour la construction des formes et contenus des discours. Je propose donc une esquisse des rapports entre objets et discours, étant entendu que ce rapport est collaboratif-contributif dans le système objets-agents humains-discours. Pour le moment je vois quatre modes de contribution témoignant de l'articulation entre les «data " des objets et les «lata » des locuteurs. Premièrement, certains objets vont afforder des genres de discours qui leur sont spécifiques ou tout du 
moins attachés. Dans le système sémiotique complexe de la décoration militaire que j'ai décrit ailleurs (Paveau 2006b), constitué de l'objet croix de guerre (objet discursif non graphique) du texte de la citation à l'ordre et de la cérémonie de remise de décoration, se propagent une série de représentations externes qui contraignent le genre épidictique. Deuxièmement, des formes morphographiques vont être partiellement explicables en termes d'affordances discursives: les listes en colonne adoptant la forme verticale du monument ou du mémorial, la disposition en étoile des noms propres sur un plan de réseau ou de ville, les monogrammes du linge de maison ou encore les formes graphiques étranges de la communication clandestine (formes stéganographiques). Troisièmement, l'objet afforde des formes particulières d'interaction verbale, de deux manières : les objets transactionnels motivent principalement l'interaction verbale car ils en sont la condition nécessaire (objets de contact comme le verre, la cigarette, le stylo, l'objet tombé, ou, de façon plus moderne, le portable ou le palm); les objets instructionnels quant à eux contraignent ou instruisent l'interaction verbale (Lahlou 2000 donne l'exemple de la machine à café au bureau, attracteur cognitif puissant qui déclenche des interactions verbales ritualisées). Enfin, certains objets affordent directement l'appel aux prédiscours des lignées discursives : par exemple la photo (de guerre, de famille, d'objet de mémoire) et d'une manière générale tous les objets-traces, qui appartiennent au domaine de la muséographie officielle ou familiale.

\section{Conclusion}

On comprend que l'approche discours et cognition déplace plusieurs frontières : «skin and skull», interne / externe, linguistique / extralinguistique, individuel / collectif. Ces déplacements sont coûteux, mais paraissent nécessaires : les disciplines texte-discours, qui ont toujours bénéficié des apports des sciences humaines et sociales, ne peuvent que s'enrtichir des avancées de la cognition sociale et culturelle.

\section{BIBLIOGRAPHIE}

Achard-Bayle G., 2005, « The "Literary Mind" and Change. Continuity and Diversity in Constructing Identities », Annual Review of Cognitive Linguistics 3 : 42-55.

Achard-Bayle G., 2006 (coord.), Pratiques 129-130, « Textes contextes ».

Althusser L., 1995 [1970], « Idéologie et appareils idéologiques d'État », dans Sur la reproduction, Paris, PUF, Actuel Marx Confrontations : 269-314.

Auroux S., 1998, La raison, le langage et les normes, Paris, PUF.

Borzeix A., Fraenkel B. (dir.), 2001, Langage et travail. Communication, cognition, action, Paris, CNRS Éditions.

Bourdieu P., 2000, «L'inconscient d'école ", Actes de la recherche en sciences sociales, 135, Paris, Seuil : 3-5. 
Boutet J., Gardin B., 2001, « Une linguistique du travail », dans Borzeix, Fraenckel (dir.) : 89-111.

Brassac C. (dir.), 2006, « Internalisme / Externalisme », Intellectica 43.

Charaudeau P., Maingueneau D. (dir.), 2002, Dictionnaire d'analyse du discours, Paris, Seuil.

Cislaru G., 2005, Étude sémantique et discursive du nom de pays dans la presse française, Thèse de doctorat, Université de Paris3-Sorbonne nouvelle.

Clark A., Chalmers D., 1998, « The extended mind », Analysis 58 (1) : 10-23.

Colletta J.-M., Nuchèze V. (de), 2002, Guide terminologique pour l'analyse des discours. Lexique des approches pragmatiques du langage, Berne, Peter Lang.

Conein B., et al. (dir.), 1981, Matérialités discursives, Lille, Presses universitaires de Lille.

Conein B., 2005, Les sens sociaux. Trois essais de sociologie cognitive, Paris, Economica.

Courtine J.-J., 1981, « Quelques problèmes théoriques et méthodologiques en analyse du discours. À propos du discours communiste adressé aux chrétiens », Langages 62 : 9-128.

Détrie C., Siblot P., Vérine B., 2001, Termes et concepts pour l'analyse du discours. Une approche praxématique, Paris, Champion.

Duranti A., 2005, « On theories and models », Discourse Studies, vol 7 (4-5) : 409-429.

Edwards D., 1997, Discourse and Cognition, London, Sage.

Edwards D., 2006, « Discourse, cognition and social practices : the rich surface of language and social interaction », Discourse Studies vol 8 (1) : 41-49.

Fitch K., 2006, « Cognitive aspects of ethnographic inquiry », Discourse Studies, vol 8 (1) : 51-57.

Flahault F., 2005, Le paradoxe de Robinson. Capitalisme et société, Paris, Mille et une nuits.

Fuchs C., 2004, Les linguistiques cognitives, Paris, Ophrys.

Giere R.N., Moffatt B., 2003, «Distributed Cognition: Where the Cognitive and the Social Merge ", Social Studies of Science 33/2:301-310.

Gilbert M., 2003, Marcher ensemble. Essais sur les fondements des phénomènes collectifs, trad. B. Auerbach et al., Paris, PUF.

Guilhaumou J., 2006, Discours et événement. L'histoire langagière des concepts, Besançon, Presses universitaires de Franche-Comté.

Halbwachs M., 1938, Esquisse d'une psychologie des classes sociales, Paris, Librairie Marcel Rivière et Cie, version numérique par J.-M. Tremblay, sur : http://www.uqac.uquebec.ca, consulté le 29.08.2006.

Havelange V., 2001, « Action cognitive et formation du lien social. Vers une réévaluation de la technique ", dans Paugam-Noisy H. et al. (dir.), La cognition entre individu et société : modèles et méthodes, Actes d'ARCO 2001, Paris, Hermès : 13-45.

Hutchins E., 1994 [1991], « Comment le cockpit se souvient de ses vitesses » (trad. de « How a Cockpit Remembers its Speed »), Sociologie du travail 4 : 461-473.

Hutchins E., 1995, Cognition in the Wild, Cambridge (MA), MIT Press.

Kara M., Privat J.-M. (coord.), 2006, Pratiques 131-132, "La littératie. Autour de Jacques Goody". Kerbrat-Orecchioni C., 1980, L'énonciation. De la subjectivité dans le langage, Paris, A. Colin.

Kerbrat-Orecchioni C., 1986, L'implicite, Paris, A. Colin. 
Lahlou S., 2000, « Attracteurs cognitifs et travail de bureau », Intellectica, Paris, 2000/1, 30 : 75-113.

Latour B., 1994, « Une sociologie sans objet ? remarque sur l'interobjectivité », Sociologie du travail 4, $\mathrm{N}^{\circ}$ spécial sur la cognition située: 587-606.

Latour B., 1994, « Note sur certains objets chevelus », Nouvelle revue d'ethnopsychiatrie 27 : 21-36.

Latour B., 1999, « When Things Strike Back a Possible Contribution of Science Studies to the Social Sciences ", British Journal of Sociology, Special Millenium Issue edited by John Urry, vol 51-1 : 105-123.

Latour B., 2001, L'espoir de Pandore, Paris, La Découverte.

Maingueneau D. (éd.), 2005, «L'analyse du discours. État de l'art et perspectives », Marges linguistiques 9 , consultable sur www.revue-texto.net.

Mazière F., 2005, L'analyse du discours. Histoire et pratiques, Paris, PUF.

Moirand S., 2003 « Les lieux d'inscription d'une mémoire interdiscursive » in Le langage des médias : des discours éphémères ?,Paris, L’Harmattan : 83-111.

Moirand S., 2004, « La circulation interdiscursive comme lieu de construction de domaines de mémoire par les médias ", in Lopez Munoz J.M. et al. (dir), Le discours rapporté dans tous ses états, Paris, L'Harmattan : 373-385.

Moirand S., 2007, Les discours de la presse quotidienne, Paris, PUF.

Norman D. A., 1993, « Les artefacts cognitifs » dans Conein B. et al. (dir.), Les objets dans l'action. De la maison au laboratoire, Paris, éd. de l'EHESS : 15-34.

Nyckees V., 1998, La Sémantique, Paris, Belin.

Nyckees V., 2003, « La perspective médiationniste en linguistique », dans M. Siksou (éd.),

Variation, construction et instrumentation du sens, Paris, Hermès : 47-72.

Paveau M.-A., 2006a, Les prédiscours. Sens, mémoire, cognition, Paris, Presses de la Sorbonne nouvelle.

Paveau M.-A., 2006b, « La citation militaire : système sémiotique, pratique honorifique », dans Lopez-Muñoz, J-M, Marnette, S., Rosier L. (éds), Dans la jungle du discours rapporté: genres de discours et discours rapporté, Presses de l'Université de Cadix, Cadix : 277-286.

Paveau M.-A., 2007a, « Les normes perceptives de la linguistique populaire », Langage et société 121, Paris, Éditions de la MSH : 93-109.

Paveau M.-A. 2007b (à par.), « Paroles antérieures. Les prédiscours entre mémoire et cognition », in Amossy R. (dir.), Revue de Philologie et linguistique portugaise , numéro spécial sur l'analyse du discours.

Paveau M.-A., Rosier L., (en cours), Pour une histoire des idées. Les analystes du discours 1968-2002, recherche FNRS par entretiens filmés auprès d'une quarantaine d'analystes du discours.

Paveau M.-A., Rosier L., 2005a, « Éléments pour une histoire de l'analyse du discours. Théories en conflit et ciment phraséologique », communication au colloque franco-allemand : «L'analyse du discours en France et en Allemagne », Créteil, Céditec, 2 Juillet, texte consultable en ligne sur http://www.johannes-angermueller.de/francais/adfa.html

Paveau M.-A., Rosier L., 2005b, « Grammaire de la liste », communication au colloque Représentations du sens linguistique 3, «Les sens en marge », Bruxelles, 3-5 novembre. 
Paveau M.-A., Rosier L., 2007 (à par.), « Le discours des objets. Pratiques et techniques de circulation entre clandestinité et exhibition discursive ", communication au colloque international Ci-dit,Circulation des discours et liens sociaux, Université Laval, Québec, 5-7. octobre.

Quéré L., 1998, « La cognition comme action incarnée », dans Borzeix A. et al. (dir.), Sociologie et connaissance. Nouvelles approches cognitives, Paris, CNRS Éditions : 143-164.

Ramognino N., Vergès P. (dir.), 2005, Sociologie et cognition sociale, Actes du colloque de septembre 2003, Aix, Publications de l'université de Provence.

Roschelle J., Teasley S.D., 1995, « Construction of shared knowledge in collaborative problemsolving ", in O'Malley C. (ed.), Computer-supported collaborative learning, New York, Springer-Verlag.

Temmar M., Bonnafous S. (dir.), 2007, Analyse du discours et sciences humaines, Paris, Ophrys.

Thévenot L., 1998, « Pragmatiques de la connaissance », dans Borzeix A. et al. (dir.), Sociologie et connaissance. Nouvelles approches cognitives, Paris, CNRS Éditions : 101-139.

Tomasello M., 2004, Aux origines de la cognition humaine, trad. Y. Bonin, Paris, Retz.

Valette M., 2006, Linguistiques énonciatives et cognitives françaises, Paris, Champion.

Van Dijk T., 1985, « Cognitive situation models in disocurse production », in Forgas J.P. (ed.), Language and social situations,New York, Springer : 61-79.

Van Dijk T., 1989, « Social cognition and discourse », in Giles H, Robinson R.P. (eds.), Handbook of social psychology and language, Chichester, Wiley : 163-183.

Van Dijk T., 1993, « Discourse and cognition in society ", in Crowley D., Mitchell D., Communication Theory Today,Oxford, Pergamon Press : 107-126.

Van Dijk T., 1997, « Cognitive context models ans discourse »in Stamenow M. (ed.), Language Structure, Discourse and the Access to Consciousness, Amsterdam, Benjamins : 189-226.

Van Dijk T. (dir.), 2006, Discourse Studies, « Discourse, interaction and cognition », Vol 8 (1).

Van Dijk T., 2006, « Discourse, context and cognition », Discourse Studies, Vol 8 (1) : 159-177.

\section{NOTES}

1. J'utilise désormais le composé texte-discours dans « disciplines texte-discours » ou " approche texte-discours» par exemple, pour nommer des approches qui portent sur les productions verbales en situation, qu'elles mettent plutôt l'accent sur la construction de la textualité ou les conditions sociales, historiques et culturelles de production. Même si ce n'est pas explicite dans mon travail qui ne porte pas spécifiquement sur ces dimensions, le terme discours englobe la conversation et l'interaction.

2. Rappelons que le discours est l'objet de multiples approches en sciences du langage et sciences de l'information et de la communication, des plus étroitement linguistiques (le discours de la sémantique théorique du discours par exemple) aux plus largement sociologiques (le discours de l'analyse de contenu), approches parcourues dans Maingueneau (dir.) 2005. Le syntagme analyse $d u$ discours, correspond, dans ma perspective, à une approche scientifique repérable dans l'histoire des sciences du langage, approche qui a ses fondateurs (années 1960, Harris, Dubois, Pêcheux), ses concepts théoriques (formation discursive, inter- et intradiscours, préconstruit, fil du discours; voir Paveau, Rosier 2005, Paveau 2006a), ses évolutions et des transformations (Guilhaumou 2006). Comme Moirand (ici-même), mon approche du discours est et veut rester 
linguistique, même informée par la cognition, et je pense « analyse linguistique du discours » ou « linguistique discursive » quand je dis analyse du discours.

3. En fait, comme le montre Chabrolle-Cerretini 2006, la notion est plus ancienne puisqu'elle est proposée par Humboldt dans les années 1820 . Nous renvoyons à ce travail et à son article icimême pour des approfondissements historiques.

4. Sur les différentes versions actuelles de l'analyse du discours et leur lecture historique et épistémologique, voir Paveau, Rosier 2005.

5. Il faut préciser que social et culturel ne s'opposent pas ici à individuel mais à interne, l'interrogation individuel $v s$ collectif étant secondaire par rapport au débat interne vs externe.

6. Clark, A. 1989. Microcognition. MIT Press - Hutchins, E. 1995. Cognition in the Wild. Cambridge, MA: MIT Press - Kirsh, D. 1995. The intelligent use of space. Artificial Intelligence 73:31-68 McClelland, J.L, D.E. Rumelhart, \& G.E. Hinton 1986. The appeal of parallel distributed processing". in McClelland \& Rumelhart, eds, Parallel Distributed Processing, Volume 2. Cambridge, MA: MIT Press.

7. Pour une synthèse voir Ramognino, Vergès (dir.) 2005.

8. Sur l'école dite française de l'analyse du discours et les ancrages politiques, idéologiques, intellectuels et scientifiques des premiers analystes du discours voir Mazière 2005 et Paveau, Rosier 2005.

9. Communication personnelle, février 2007.

10. Pour Gilbert, un groupe d'individus forment un « sujet pluriel » (plural subject) quand ils sont conjointement engagés à faire quelque chose (marcher ensemble, tenir une conversation, etc.), comme s'ils formaient un seul corps (as a body).

11. Pease A. \& B., 1999-2002, Why Women Can't Read Map and Won't Stop Talking, Men Don't Listen And Can Only Do One Thing At A Time, Why men Lie and Woman Cry, trad. française : Pourquoi les hommes... Pourquoi les femmes..., Paris, First Éditions.

12. Dès les années 1920 Halbwachs, contre la psychologie individualiste dominante à l'époque (Blondel), pose la nature collective des phénomènes intuitivement considérés comme les plus individuels : rêve, sentiments divers, manières d'être. Voir en particulier Halbwachs 1938.

13. Bien représentée en France par les travaux de Bourdieu et de ses continuateurs (Baudelot, Champagne, Sapiro, Passeron, Wacquant).

14. «Le système des schèmes cognitifs qui sont au principe de la construction de la réalité et qui sont communs à l'ensemble d'une société, à un moment donné, constitue l'inconscient culturel, ou mieux, le transcendantal historique qui fonde le sens commun (ou la doxa), c'est-à-dire tout ce qui est taken for granted, qui va de soi, qui va sans dire " (Bourdieu 2000:3).

15. L'étude des artefacts est ancienne, que ce soit chez Wundt dans les années 1910, dans l'école socio-historique russe avec Leontiev, Luria et Vygotski bien sûr. Un ensemble de travaux en Scandinavie, Union soviétique et Allemagne se penche pendant une bonne partie du XXe siècle sur la «théorie de l'activité ». Les sciences du langage ont très modérément intégré ces perspectives. Du côté de l'anthropologie de l'écriture et du monde anglo-saxon, on ne peut que mentionner le travail de Goody sur la dimension technique et technologique de l'écrit. Mais la prise en compte de la scripturalité débouche rarement sur la prise en compte des outils et artefacts en tant qu'objets non humains, sauf exception hors du champ de la linguistique (l'anthropologue Dardy par exemple). 


\section{RÉSUMÉS}

L'analyse du discours est à la recherche d'un nouveau souffle théorique. De leur côté les sciences cognitives ont depuis les années 1980-1990 intégré les questions du contexte et de la culture et s'ancrent actuellement, en France tout du moins, dans une histoire intellectuelle qui reconnaît désormais la place faite aux activités mentales dans la modélisation de la production langagière. La rencontre semble inévitable et sur le terrain encore étroit et nouveau que constitue cette articulation, certaines recherches tentent de rendre compte de la dimension cognitive dans le discursif et inversement. Plusieurs directions se dessinent en France actuellement, et parmi elles une articulation discours-cognition mettant à contribution la phénoménologie de la perception et la cognition sociale. L'analyse du discours connaît en effet actuellement un tournant cognitif reposant sur le réexamen de la question du contexte dans les disciplines texte-discours. L'appel aux données de la cognition, parce qu'il oblige le chercheur à repenser la notion de contexte, semble en effet à même de fournir des éléments et des regards nouveaux sur cette réalité complexe. Le domaine "discourse and cognition» est représenté par la théorie des «mental models» (Van Dijk) qui constitue une version faible de la cognition dans le cadre d'une "sociocognitive approach of discourse». Nous proposons une version plus robuste s'inspirant des approches de la cognition sociale qui reposent sur des choix théoriques concernant la nature de l'esprit et la définition du contexte comme environnement matériel. Cette perspective est fondée sur l'idée d'une cognition externe (extended mind), dans le cadre du paradigme externaliste, postulant l'extériorité de l'esprit, dont la version la plus séduisante pour notre propos est l'hypothèse de l'« active externalism » de Clark et Chalmers. Nous proposons à partir de là pour l'analyse du discours une conception du contexte comme continuum entre interne et externe : le contexte intègre les données environnementales qui se constituent à la fois de cadres internes (les prédiscours comme cadres de savoirs, de croyances et de pratiques) eux-mêmes informés par les données externes, et des réalités extérieures de notre environnement matériel concret (décors naturels ou artificiels, espaces, objets culturels et techniques, artefacts, supports, etc.). Nous définissons, dans la perspective de la cognition sociale, les prédiscours comme des données propagées par une mémoire cognitivo-discursive, résultat d'un retravail du concept de "mémoire discursive» et distribuées par un certain nombre d'agents internes et externes comme les artefacts ou les technologies cognitives. Choisissant de parler de distribution plus que de partage, nous optons pour une version dynamique de la collectivité, où c'est l'individu luimême qui est collectif, et qui appartient à un ensemble où s'articulent agents humains et non humains : c'est la mémoire du cockpit défendue par Hutchins. Dans le cadre d'une telle approche, relevant de la cognition distribuée, nous prenons en compte les éléments objectaux et techniques dans le processus de construction des discours. Nous proposons alors l'idée d'une technologie discursive pour désigner des techniques, artefacts ou objets qui configurent, formatent, et informent les prédiscours en vue de l'élaboration des discours. Outils linguistiques et discursifs (artefacts, objets, etc.) constituent alors des contributeurs cognitifs au discours et doivent être pris en compte dans les théories méthodes d'analyse du discours.

While discourse analysis seeks a new theoretical lease of life, cognitive sciences have integrated the questions of context and culture. Certain researches attempt to give an account of the cognitive dimension in the discursive, and contrariwise. The "discourse and cognition" domain is 
currently represented by Van Dijk's “mental models" theory in the English-speaking world. However, works are emerging in France, among them a discourse-cognition articulation adding a contribution from the phenomenology of perception and social cognition. Here, we propose a discourse-cognition articulation inspired by the social cognition approaches based on theoretical choices concerning the nature of the spirit and the definition of the context as a material environment. This perspective is based on the idea of an external cognition, the most attractive version of which, for our purpose, is the Clark and Chalmers hypothesis of "active externalism". From this, we propose a conception of context as a continuum, integrating environmental data consisting both of internal frameworks (pre-discourses as frameworks of knowledge, beliefs and practices) themselves informed by external data, and the exterior realities of our material, concrete environment. Within such an approach, deriving from distributed cognition, we take into account objectal elements and techniques in the process of discourse construction. So, we propose the idea of a discursive technology to designate techniques, artefacts or objects which configure, format, and inform pre-discourses with a view to elaborating discourses.

\section{INDEX}

Mots-clés : analyse du discours, cognition distribuée, contexte, externalisme, extended mind, outils discursifs, outils linguistiques, prédiscours, technologie discursive

Keywords : discourse analysis, distributed cognition, context, externalism, discursive tools, linguistic tools, pre-discourse, discursive technology

\section{AUTEUR}

\section{MARIE-ANNE PAVEAU}

Université Paris 13, Cenel 